\title{
Diseño Mecatrónico de un Robot de Tres Grados de Libertad Aplicando la Metodología de Nigel Cross
}

\author{
Mechatronic Design of a Robot Three Degrees of Freedom Applying the Nigel Cross \\ Methodology \\ Irving Morgado-González ${ }^{a}$, Martín Ortiz-Domínguez $^{b}$, Arturo Cruz-Avilés ${ }^{c}$, Miguel A. \\ Paredes-Rueda ${ }^{d}$, José A. García-Macedo ${ }^{e}$, Rodolfo Velázquez-Mancilla ${ }^{f}$, Miguel A. Flores- \\ Rentería $^{g}$,Jorge Zuno-Silva $^{h}$, José M. Farfán-García $^{i}$
}

\begin{abstract}
:
This article describes the important aspects of the methodology of design, manufacturing and the kinematic modeling of a robot manipulator of three degrees of freedom are described. The robot manipulator will be used for educational and research purposes in the areas of robotics in the Mechanical Engineering Undergraduate Program at the Escuela Superior de Ciudad Sahagún-UAEH. We present a proposal of a design of a robotic manipulator with 5-bars, similar to ABB's IRB1400. Likewise, gearmotors with quadrature encoders are used and an Arduino card is used as data acquisition. The direct and inverse kinematics is calculated with the help of the Denavit-Hartenberg parameters. Simulation tests of the behavior of the robot and the validation of the kinematic model are carried out.
\end{abstract}

\section{Keywords:}

Robotics, robotic manipulator, Kinematics of manipulators, Nigel Cross.

\section{Resumen:}

En el presente artículo se describen los aspectos importantes de la metodología de diseño, la fabricación y el modelado cinemático de un robot manipulador de tres grados de libertad. El manipulador robótico diseñado tendrá fines educativos e investigación y será utilizado en el área de robótica en la Escuela Superior de Ciudad Sahagún-UAEH. Se presenta la propuesta de diseño de un robot manipulador de 5 barras similar al IRB1400 de ABB. Se utilizan motoreductores con encoders de cuadratura y como adquisición de datos una tarjeta Arduino. Se calcula la cinemática directa e inversa del manipulador, mediante el uso de los parámetros de DenavitHartenberg. Se realizan pruebas de simulación del comportamiento del robot y la validación del modelo cinemático.

\footnotetext{
a Estudiante de Doctorado, Universidad Autónoma del Estado de Hidalgo-AACTyM, Mineral de la Reforma, Hidalgo, México, Email: imorgadog@hotmail.com;

b Autor de Correspondencia, Licenciatura en Ingeniería Mecánica, Escuela Superior de Ciudad Sahagún, UAEH, Tepeapulco, Hidalgo, México, ORCID: 0000-0003-4475-9804, Email: martin_ortiz@uaeh.edu.mx;

c Licenciatura en Ingeniería Mecánica, Escuela Superior de Ciudad Sahagún, UAEH, Tepeapulco, Hidalgo, México, ORCID: 0000-00030455-1646, Email: arturo_cruz8085@uaeh.edu.mx;

d División de estudios de posgrado e investigación, Instituto Tecnológico de Tlalnepantla-ITTLA, Tlalnepantla de Baz, Estado de México, México, Email: mparedes@ittla.edu.mx;

e Área de ingeniería y ciencias, Tecnológico de Monterrey-Campus Estado de México, Ciudad de México, México, josegarc@itesm.mx; ${ }^{\mathrm{f}}$ Licenciatura en Ingeniería de Mecánica, Instituto Tecnológico de Tlalnepantla-ITTLA, Tlalnepantla de Baz, Estado de México, México, Email: itladepi20@hotmail.com

g Licenciatura en Ingeniería Mecánica, Escuela Superior de Ciudad Sahagún, UAEH, Tepeapulco, Hidalgo, México, Email: miguel_flores10233@uaeh.edu.mx;

h Director, Escuela Superior de Ciudad Sahagún, UAEH, Tepeapulco, Hidalgo, México, zunojorge@gmail.com;

i Licenciatura en ingeniería mecánica, Universidad Politécnica de Pachuca, Zempoala, Hidalgo, México, Email: jmfarfang@gmail.com;
} 


\section{Palabras Clave:}

Robótica, manipulador robótico, cinemática de manipuladores, Nigel Cross

\section{Introducción}

El presente trabajo describe los aspectos principales a la hora de diseñar, ya sea un prototipo, un producto nuevo o la mejora de algo existente. El enfoque organizado y ordenado para resolver problemas se conoce como proceso de diseño, el cual en ingeniería toma en cuenta las necesidades, los deseos y los problemas de la sociedad mediante la aplicación de principios científicos, experiencia y creatividad. Los diferentes tipos de dibujos técnicos tienen una función específica en el proceso de diseño de ingeniería, es decir los bosquejos a mano alzada capturan y documentan el proceso de ideación. Mientras los modelos y dibujos CAD (Computer-aided design por sus siglas en inglés) capturan el diseño y especifican los detalles necesarios para su fabricación. El proceso de diseño para cualquier producto requiere una comprensión clara de las funciones y el requerimiento esperado de ese producto. Se estima que entre 70 y $80 \%$ de los costos de desarrollo y fabricación de los productos se determina durante las fases de diseño inicial. 2

Al diseñar es necesario imaginar la forma en que va a funcionar el aparato o máquina. La idea principal es tener un robot manipulador con movimientos articulares, debido a que son los que se encuentran mayormente en las industrias. Adueza L y Aguirre [3] proponen un diseño de un robot manipulador de tres grados de libertad, mostrando los requerimientos del robot a diseñar, sus componentes empleados para el proceso de fabricación, selección de actuadores, sensores, y el tipo de transmisión de potencia, adecuados para su correcto funcionamiento tomando en cuenta las características de los robots manipuladores comerciales. Para la creación de un nuevo producto existen varios modelos para el proceso de diseño, el modelo simple de 4 etapas y el modelo francés son un par de ejemplos. Estos modelos dan paso a distintos métodos, uno de los métodos más utilizados es el creativo, en el que el diseñador recurre a su creatividad e imaginación, a analogías de sistemas o productos que no son afines, etc. Otro método es el racional, el cual se describe por Nigel Cross y es el utilizado esta ocasión. 1

Al diseñar es necesario imaginar la forma en que va a funcionar el aparato o máquina. La idea principal es tener un robot manipulador con movimientos articulares, debido a que son los que se encuentran mayormente en las industrias. Adueza L y Aguirre proponen un diseño de un robot manipulador de tres grados de libertad, mostrando los requerimientos del robot a diseñar, sus componentes empleados para el proceso de fabricación, selección de actuadores, sensores, y el tipo de transmisión de potencia, adecuados para su correcto funcionamiento el que el diseñador recurre a su creatividad e imaginación, a analogías de sistemas o productos que no son afines, etc. Otro método es el racional, el cual se describe por Nigel Cross y es el utilizado esta ocasión. 1,3

Para el desarrollo del proyecto, se llevaron a cabo las 7 etapas de la metodología de diseño de Nigel Cross. A continuación, se describen estas etapas.

\section{A. Clarificar Objetivos}

En esta etapa se parte del objetivo general que es "Diseñar un robot manipulador de tres grados de libertad para fines de investigación". El robot a diseñar es del tipo manipulador robótico industrial similar a la arquitectura del IRB 1400 de ABB, inicialmente será diseñado para que cumpla funciones de posicionamiento, a medida que avance la investigación se irá añadiendo la capacidad de carga, descarga, manipulación y traslado de objetos. A partir del conocimiento de las necesidades del usuario final, se divide este objetivo en sub-objetivos como se muestra en Figura 1.

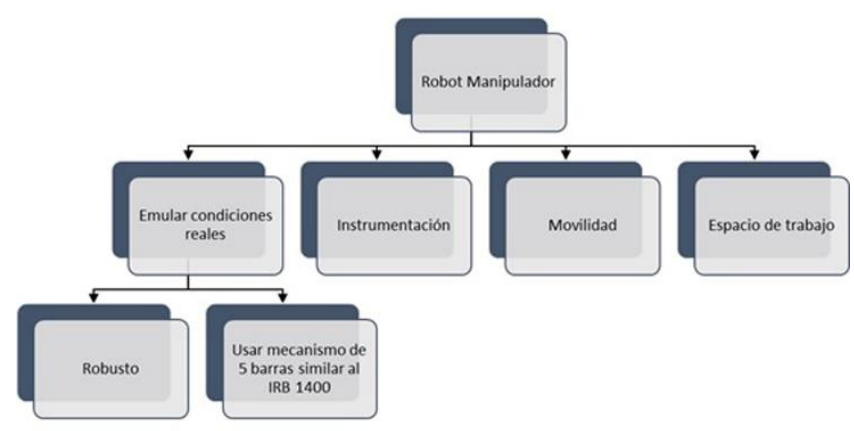

Figura 1. Árbol de objetivos.

(Elaboración Propia)

Se puede observar cuatro sub-objetivos principales, la primera es emular condiciones reales de movimiento del robot, la segunda es tener evidencia del movimiento realizado por el mecanismo del robot mediante un sistema de instrumentación, la tercera es darle movilidad, que no 
requiera de una instalación propia y específica para su funcionamiento, por último, la cuarta necesidad es permitir que el efector-final del robot manipulador tenga un espacio de trabajo adecuado para realizar ejercicios de posicionamiento espacial.

\section{B. Establecer Funciones}

Para establecer las funciones utilizamos el método de análisis de funciones del cual se obtiene un modelo del sistema tipo caja transparente que se muestra en la Figura 2, describiendo sus entradas, sub-funciones y salidas. Esta descripción gráfica parte de las necesidades del producto y los resultados esperados y sirve para identificar el comportamiento del sistema mediante funciones específicas de una manera simplificada, entre más simple se defina el problema es más fácil obtener una solución.

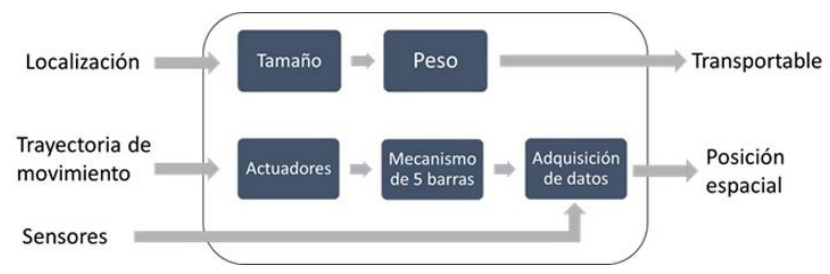

Figura 2. Modelo del sistema (caja transparente).

(Elaboración Propia)

\section{ConFigurar Requerimientos}

En esta etapa se define qué se requiere del producto y qué desempeño se espera de él. En la tabla 1 se muestran los requerimientos.

\begin{tabular}{c|c}
\hline & \multicolumn{2}{c}{ Estructura } \\
\hline Tamaño & Menor a 1 mts. \\
Peso & Menor a 15 Kg. \\
Material & Aluminio, Nylamid, Nylacero \\
Movimiento & Fijo en operación-Móvil para transporte \\
\hline & Sistema Electrónico \\
\hline Interfaz & Gráfica e Intuitiva \\
Paro de Emergencia & Fisico y en la interfaz \\
Alimentación & Eléctrica (120 Vca, 12Vcd) \\
Sensores & Posición, Limite \\
\hline
\end{tabular}

Tabla 1. Requerimientos del producto.

(Elaboración Propia)

Los componentes mostrados en la tabla anterior se basan principalmente en la estructura física y el sistema electrónico que es con lo que debe contar el robot manipulador, los requerimientos se establecen dentro de ciertos límites, es decir, uno de los límites más importantes, por mencionar uno, es el costo, que no es más que lo que el cliente está dispuesto a gastar en algo nuevo o lo que se espera que el cliente pague como precio de compra de un producto.

\section{Determinar Características}

Para determinar las características del producto se recurre al método del despliegue de la función de calidad. Primero se identifica qué requiere el cliente y se compara con los requerimientos de ingeniería que implican. Estos requerimientos se colocan en una tabla comparativa donde se pueda observar la relación del requerimiento con el proceso de ingeniería como se muestran en la Figura 3.

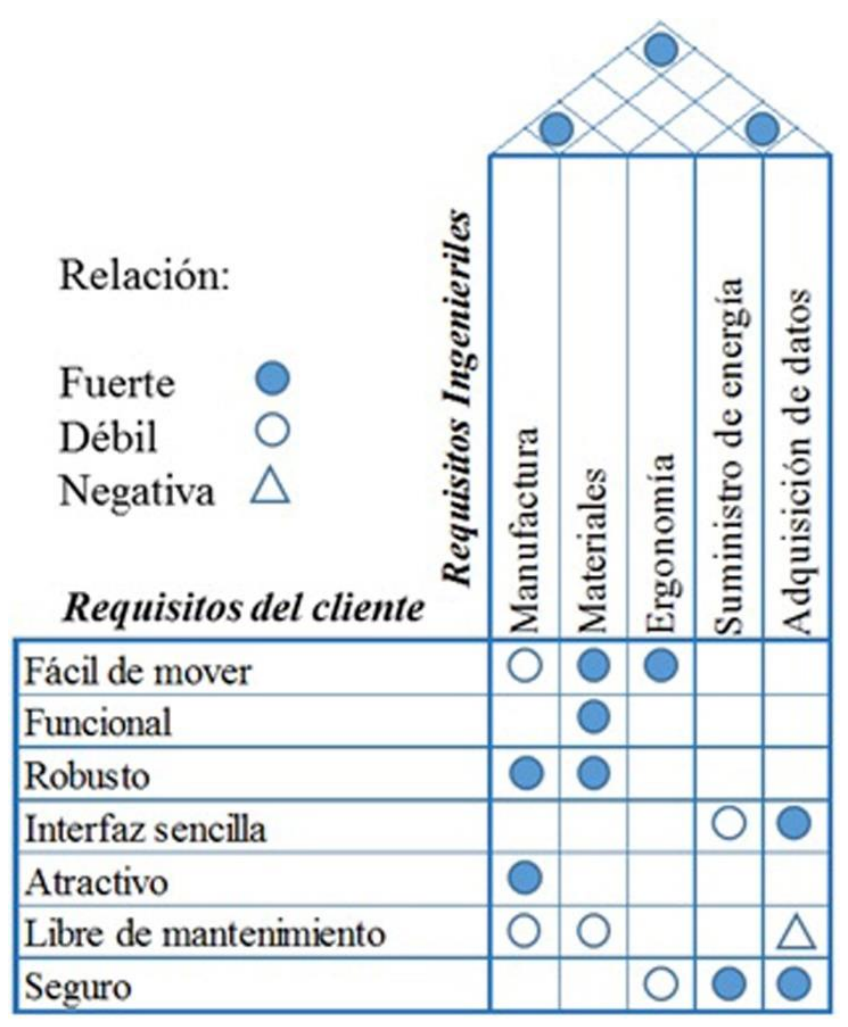

Figura 3. Despliegue de la función de calidad.

(Elaboración Propia)

Con esto se determina que el producto debe tener las siguientes funciones:

- Fijación y movilidad. El producto debe poder fijarse en una mesa de trabajo para evitar vibraciones y movimientos indeseados, y debe también tener la movilidad para poder almacenar el robot cuando no se utilice o poder cambiarlo de mesa de trabajo según se requiera.

- Interfaz gráfica. Se requiere una interfaz amigable para que el operador pueda visualizar los 
movimientos, y estados de cada una de las articulaciones del robot.

- Estructura robusta. Se requiere de una estructura que no tenga deformaciones y soporte de trabajo continuo.

- Sensores. Son necesarios para activar o desactivar el robot, y obtener las mediciones de cada una de las articulaciones del robot.

\section{E. Generar Alternativas}

Para generar múltiples alternativas posibles del producto se crea una gráfica en la que se proponen alternativas para cada función o característica que integra al producto final. En la tabla 2 se muestran las diferentes soluciones para las sub-funciones. Una solución se puede formar también a partir de la combinación de dos soluciones propuestas.

\begin{tabular}{|c|c|c|c|}
\hline Solución & 1 \\
\hline $\begin{array}{l}\text { Transmisión } \\
\text { de potencia }\end{array}$ \\
\hline Estructura \\
\hline Rodamientos
\end{tabular}

Tabla 2. Gráfica morfológica.

(Elaboración Propia)

Para la primera sub-función que son los motores, existen cuatro propuestas para seleccionar el tipo de actuador que tendrán las articulaciones rotacionales, la primera es el motor sin escobillas (brushless) de corriente directa, de poco peso y apropiado para prototipos, el segundo un motor a pasos, con alto torque, el tercero motor de corriente directa $(C D)$ de imán permanente, el cuarto un motor de corriente alterna (CA) de jaula de ardilla. Para la transmisión de potencia, el movimiento a realizar por las articulaciones es del tipo de transmisión rotacionalrotacional, para este tipo de transmisión existen cuatro soluciones, la primera es por banda síncrona, que nos permite transmitir potencia a una relación constante de velocidad angular, la segunda es por banda de tipo $V$ que se pueden emplear para distancias grandes entre centros, la tercera es por cadena y catrina, este tipo de transmisión no tiene problemas de deslizamiento ni de arrastre, la cuarta solución es un sistema de engranaje que nos permitirá ampliar la capacidad limitada del par en el motor y reducir la velocidad rotacional del mismo.

En la estructura mecánica del robot se tienen cuatro propuestas de solución, la primera es muy común utilizar placa de aluminio 6061 que tiene excelente resistencia a la corrosión, buena maquinabilidad, y se suelda satisfactoriamente, la segunda opción es barra de aluminio, la tercera opción cold rolled, que cuenta con excelentes propiedades mecánicas tales como dureza del material y su resistencia a la tensión, por último la cuarta opción es tubular de nylamid $\mathrm{M}$, que resulta de dos a ocho veces más ligero que los metales, tiene resistencia al impacto y resistencia dieléctrica. En los rodamientos se tiene cuatro propuestas de solución para las articulaciones del robot, la primera es utilizar rótulas de esfera hueca, la segunda es un rodamiento de bolas de carga axial, la tercera opción un rodamiento cónico para soportar ambas cargas, (axial y radial), y por último la cuarta alternativa es un rodamiento de bolas de carga radial.

Los sensores de entrada se tienen tres propuestas para medir la posición articular de cada uno de las articulaciones del motor, la primera es un resolver, la segunda es un encoder óptico, y por último la tercera opción es un potenciómetro. Para la movilidad y fijación se tienen igualmente tres alternativas distintas, la primera es ser movido por una o dos personas, la segunda es un montacargas de motor y el tercero un montacargas manual que puede ser llevado por una o dos personas. Finalmente está la interfaz, la primera opción es utilizar el lenguaje de programación LabVIEW $^{\circledR}$ para crear la interfaz gráfica y una tarjeta de adquisición de la marca National Instruments ${ }^{\circledR}$ para la instrumentación. La segunda opción es realizar una interfaz en Java® y utilizar una tarjeta electrónica Arduino ${ }^{\circledR}$ para realizar la operación del mecanismo y la adquisición de datos. Por último, la tercera opción, la interfaz sería programada en Matlab ${ }^{\circledR}$ y utilizar una tarjeta electrónica Arduino ${ }^{\circledR}$.

\section{F. Evaluar Alternativas}


Para evaluar las alternativas, Nigel Cross propone crear una tabla comparativa, jerarquizar las funciones y asignarles una ponderación, En lugar de eso, en este paso, basándose en la información recopilada anteriormente, se evalúan las soluciones posibles para obtener una solución general, y un diseño final. Esta evaluación se hace a criterio del diseñador, considerando cada una de las cualidades que posee cada alternativa de solución. En las siguientes tablas se muestran las evaluaciones para cada sub-función, del lado izquierdo están los puntos a evaluar, en la primera columna se cuantifica la ponderación de importancia de cada punto a evaluar, y en las siguientes columnas se encuentra la evaluación de cada solución propuesta, para obtener la suma de puntos se multiplica el valor de cada punto por su ponderación de importancia y se suman verticalmente. Esta es una forma de dar un valor cuantitativo a cada alternativa considerando las cualidades de cada una. 1

En la tabla 3 se muestra la evaluación de la sub-función "Motores" de la cual se selecciona la solución uno correspondiente a motor de corriente directa.

\begin{tabular}{c|ccccc}
\hline Aspectos a evaluar & Ponderación & Solución 1 & Solución 2 & Solución 3 & Solución 4 \\
\hline Consumo de energía & 0.7 & 8 & 7 & 7 & 6 \\
Dimensiones & 0.6 & 9 & 6 & 6 & 5 \\
Manipulación & 0.8 & 9 & 8 & 8 & 7 \\
Costo & 1 & 8 & 7 & 7 & 7 \\
\hline Total & & 26.2 & 21,9 & 21,9 & 19.8 \\
\hline
\end{tabular}

Tabla 3. Evaluación de alternativas: sub-función "Motores".

(Elaboración Propia)

En la tabla 4 se muestra la evaluación de la sub-función "Transmisión de potencia" de la cual se selecciona la cuarta solución que es un tren de engranes de reducción puesto que el motor cuenta con una transmisión de engranes acoplada.

\begin{tabular}{c|ccccc}
\hline Aspectos a evaluar & Ponderación & Solución 1 & Solución 2 & Solución 3 & Solucion 4 \\
\hline Dimensiones & 0.7 & 6 & 6 & 6 & 8 \\
Mantenimiento & 0.8 & 6 & 6 & 5 & 9 \\
Ruido & 0.9 & 6 & 6 & 4 & 9 \\
Costo & 0.6 & 5 & 5 & 5 & 7 \\
\hline Total & & 17.4 & 17.4 & 14.8 & 25.1 \\
\hline
\end{tabular}

Tabla 4. Evaluación de alternativas: sub-función "Transmisión de potencia".

(Elaboración Propia)

En la tabla 5 se muestra la evaluación de la sub-función "Estructura" de la cual se hace una fusión de la solución uno y dos para la construcción de la base, mientras que la solución 3 se utilizó para el diseño de los ejes y la solución cuatro, nylamid, se empleó para la fabricación del resto robot ya que cumple con los requerimientos apropiados de poco peso.

\begin{tabular}{c|cccccc}
\hline Aspectos a evaluar & Ponderacion & Solucion 1 & Solución 2 & Solución 3 & Solucion 4 \\
\hline Maquinado & 0.9 & 7 & 7 & 7 & 9 \\
Peso & 1 & 8 & 8 & 6 & 9 \\
Materiales & 0.8 & 8 & 8 & 7 & 8 \\
Costo & 0.7 & 5 & 5 & 7 & 8 \\
\hline Total & & 24.2 & 24.2 & 22.8 & 29.1 \\
\hline
\end{tabular}

Tabla 5. Evaluación de alternativas: sub-función "Estructura".

\section{(Elaboración Propia)}

En la tabla 6 se muestra la evaluación de la sub-función "Rodamientos" de la cual se hace una fusión de la solución uno, dos y cuatro; debido a su utilidad en cada una de las articulaciones del robot manipulador, los cuales son: cintura, hombro y codo.

\begin{tabular}{c|ccccc}
\hline Aspectos a evaluar & Ponderación & Solución 1 & Solución 2 & Solución 3 & Solución 4 \\
\hline Dimensiones & 0.5 & 8 & 8 & 6 & 8 \\
Duración & 1 & 8 & 8 & 8 & 8 \\
Reemplazo & 0.9 & 7 & 9 & 5 & 7 \\
Costos & 0.8 & 7 & 9 & 5 & 7 \\
\hline Total & & 23.9 & 27.3 & 19.5 & 23.9 \\
\hline
\end{tabular}

Tabla 6. Evaluación de alternativas: sub-función "Rodamientos".

$$
\text { (Elaboración Propia) }
$$

En la tabla 7 se muestra la evaluación de la sub-función "Sensores" de la cual se selecciona la solución 2 , siendo ésta el encoder óptico para medir la posición articular ya que viene integrado con los motores $C D$ seleccionados previamente en la tabla 3.

\begin{tabular}{c|cccc}
\hline Aspectos a evaluar & Ponderación & Solución 1 & Solución 2 & Solución 3 \\
\hline Rango dinámico & 0.6 & 9 & 8 & 5 \\
Resolución & 0.9 & 9 & 9 & 5 \\
Estabilidad térmica & 0.8 & 9 & 9 & 5 \\
Costo & 0.7 & 3 & 9 & 10 \\
\hline Total & & 22.8 & 26.4 & 18.5 \\
\hline
\end{tabular}

Tabla 7. Evaluación de alternativas: sub-función "Sensores".

$$
\text { (Elaboración Propia) }
$$

En la tabla 8 se muestra la evaluación de la sub-función "Movilidad" de la cual se decide la solución 1, ya que es posible transportar el robot por una o dos personas sin problemas.

\begin{tabular}{c|cccc}
\hline Aspectos a evaluar & Ponderacion & Solución 1 & Solución 2 & Solución 3 \\
\hline Inversión de tiempo & 0.8 & 8 & 6 & 6 \\
Practicidad & 0.6 & 8 & 5 & 7 \\
Costo & 0.8 & 10 & 5 & 7 \\
\hline Total & & 19.2 & 11.8 & 14.6 \\
\hline
\end{tabular}

Tabla 8. Evaluación de alternativas: sub-función "Movilidad". 
(Elaboración Propia)

Por último, en la tabla 9 se muestra la evaluación de la sub-función "Interfaz" de la cual se selecciona la tercera solución, por lo tanto, se tendrá una interfaz elaborada en Matlab $\AA$ con una tarjeta Arduino utilizada como tarjeta de adquisición de datos, debido a que tiene las características necesarias y suficientes para el propósito requerido.

\begin{tabular}{c|cccc}
\hline Aspectos a evaluar & Ponderacion & Solucion 1 & Solución 2 & Solución 3 \\
\hline Manufactura & 0.5 & 7 & 7 & 9 \\
Cantidad de material & 0.5 & 8 & 6 & 8 \\
Practicidad de uso & 0.8 & 8 & 4 & 6 \\
Costo & 0.6 & 6 & 9 & 8 \\
\hline Total & & 17.5 & 15.1 & 18.1 \\
\hline
\end{tabular}

Tabla 9. Evaluación de alternativas: sub-función "Interfaz".

(Elaboración Propia)

\section{G. Diseño conceptual}

Una vez seleccionado las alternativas más idóneas, se procede a realizar el diseño del producto teniendo las especificaciones y características mucho más claras.

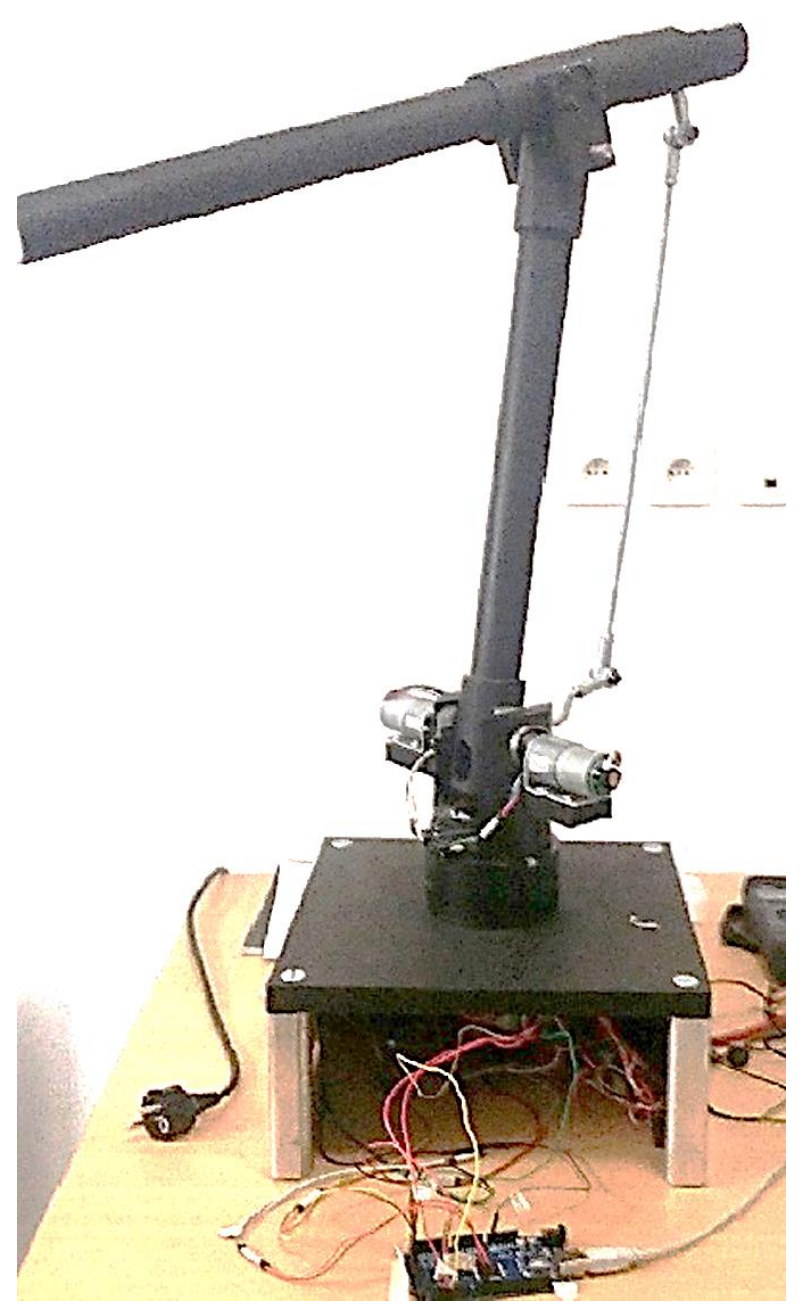

Figura 4. Prototipo del robotmanipulador.

(Elaboración Propia)

Se obtiene un prototipo final del robot manipulador, (ver Figura 4). El diseño del producto que se muestra en la Figura 5, en color verde (Placa Nylacero) y en color carmín (Placa de Aluminio 6061) se muestra la base de todo el robot (Cintura), los eslabones del robot (Nylamid tubular), se muestran en color amarillo y azul que son correspondientes al hombro, ante-brazo y brazo. Toda la estructura del robot manipulador tendrá un peso de 9.75 $\mathrm{kg}$. 


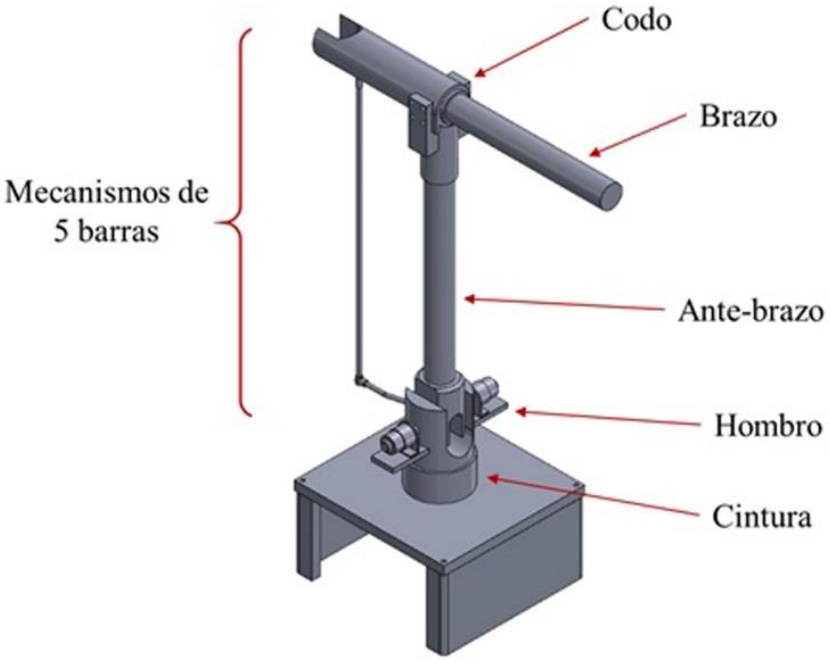

Figura 5. Diseño conceptual.

(Elaboración Propia)

La arquitectura del brazo mostrada en la Figura 5, representa una configuración de un mecanismo de 5 barras, el cual está conformada por una geometría de un paralelogramo. El proceso de operación e instrumentación realizado se basa en una interfaz programada en Matlab ${ }^{\circledR}$, con comunicación con la tarjeta Arduino, dicha interfaz trabaja según la configuración que establezca el operador, recibe la señal de los sensores utilizando la tarjeta Arduino como tarjeta de adquisición de datos que procesa la información enviándola a Matlab para mostrarlos mediante un gráfico y activando los actuadores, se almacena la información en un archivo con extensión (.xls) para su análisis. Este proceso se muestra en la Figura 6.

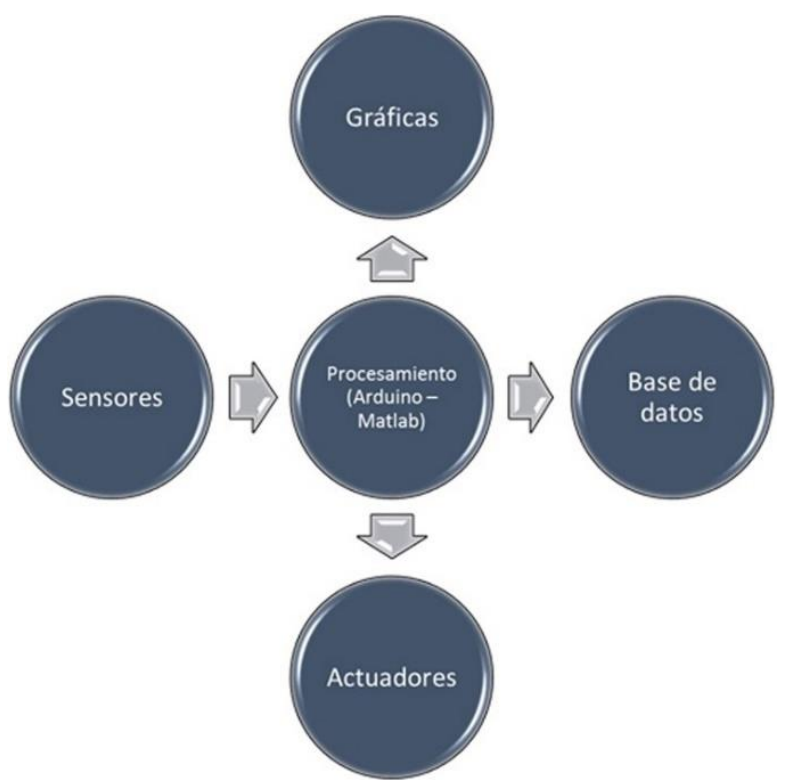

Figura 6. Diagrama de flujo: Instrumentación.

(Elaboración Propia)

\section{Modelo cinemático}

El estudio de la cinemática directa de robots manipuladores industriales proporciona elementos para analizar y diseñar el desplazamiento de trayectorias del robot, así como la orientación del efector-final (herramienta) de trabajo. La posición y orientación del efector-final del robot no se mide directamente, sino que en su lugar se calculan utilizando las lecturas de posición articular de la cinemática del robot. La cinemática inversa se utiliza para obtener las posiciones articulares necesarias que debe adoptar el robot para alcanzar la posición y la orientación del efector-final deseada. 4,5

En la Figura 7 se muestra la estructura antropomórfica, conjunto de tres articulaciones de revolución con la primera articulación ortogonal a las otras dos que son paralelas, del cual se obtiene el modelo cinemático del robot. Denavit-Hartenberg crearon un algoritmo que permite la obtención del modelo cinemático directo, el cual es un método matricial sistemático que permite establecer sistemas de coordenadas ligados a cada eslabón de un mecanismo, para así determinar la cinemática completa del mismo. Los parámetros de Denavit-Hartenberg y los límites de rango de cada una de las articulaciones se muestran en las Tablas X y XI. Los marcos representados y parámetros asociados fueron encontrados usando la convención de Craig. 4, 6

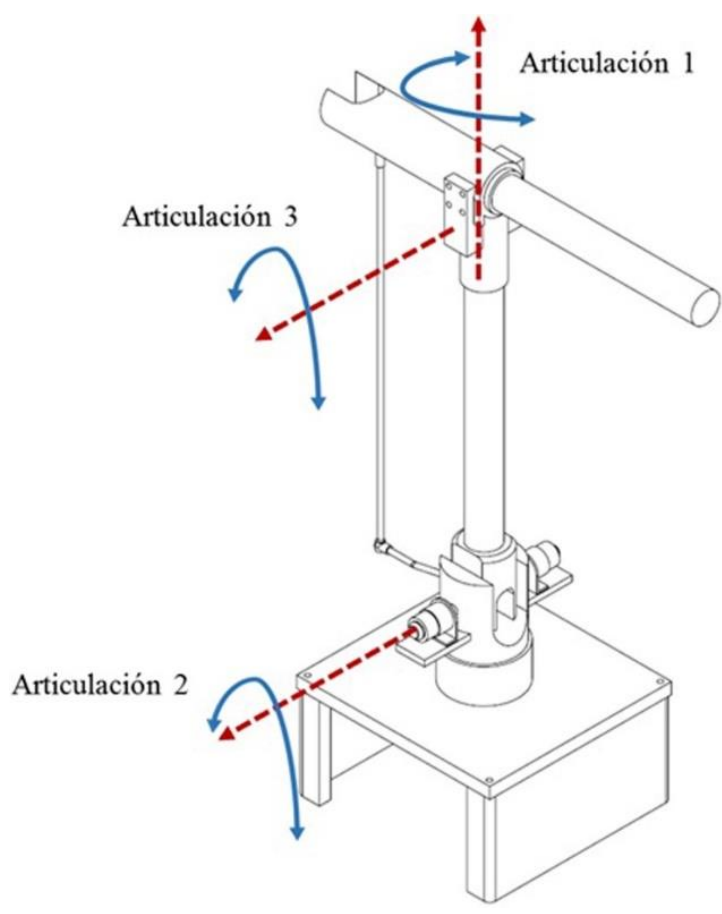

Figura 7. Estructura antropomórfica del robot "Clony" (Elaboración Propia) 


\begin{tabular}{c|cccc}
\hline Eslabón & $\theta_{i}\left({ }^{\circ}\right)$ & $\alpha_{i-1}\left({ }^{\circ}\right)$ & $a_{i-1}(m m)$ & $d_{i}(\mathrm{~mm})$ \\
\hline $\mathbf{1}$ & $\theta_{1}\left(0^{\circ}\right)$ & $0^{\circ}$ & 0 & $d_{1}$ \\
$\mathbf{2}$ & $\theta_{2}\left(-90^{\circ}\right)$ & $-90^{\circ}$ & 0 & 0 \\
3 & $\theta_{3}\left(0^{\circ}\right)$ & $0^{\circ}$ & $l_{1}$ & 0 \\
$\mathbf{4}$ & $\theta_{4}\left(0^{\circ}\right)$ & $-90^{\circ}$ & $d$ & $l_{4}$ \\
\hline
\end{tabular}

Tabla 10. Parámetros Denavit-Hartenberg del manipulador diseñado.

(Elaboración Propia)

\begin{tabular}{c|c}
\hline Eslabón & Espacio de trabajo (9) \\
\hline $\mathbf{1}$ & $+130^{\circ} a-130^{\circ}$ \\
$\mathbf{2}$ & $+50^{\circ} a-50^{\circ}$ \\
$\mathbf{3}$ & $+90^{\circ} a-30^{\circ}$ \\
\hline
\end{tabular}

Tabla 11. Rango de operación del robot manipulador. (Elaboración Propia)

\section{Cinemática directa de posición}

El problema cinemático directo de posición se obtiene en términos de encontrar una matriz de transformación que relaciona el sistema de coordenadas de cada eslabón en movimiento respecto a un sistema de coordenadas que se toma como referencia. En esta representación se usan las matrices de transformación homogénea de dimensiones de $4 \times 4$, la cual incluye operaciones de traslación y de orientación. La cinemática directa de posición para el robot manipulador propuesto se obtiene del centro de masa de cada uno de los eslabones usando la definición de matriz de transformación homogénea mostrada en la Ec. (1). 4

$$
\begin{gathered}
T_{i}^{i-1}=\left[\begin{array}{cccc}
c_{i} & -s_{i} & 0 & a_{i-1} \\
s_{i} c \alpha_{i-1} & c_{i} c \alpha_{i-1} & -s \alpha_{i-1} & -s \alpha_{i-1} d_{i} \\
s_{i} s \alpha_{i-1} & c_{i} s \alpha_{i-1} & c \alpha_{i-1} & c \alpha_{i-1} d_{i} \\
0 & 0 & 0 & 1
\end{array}\right] \\
P_{1}=\left[\begin{array}{c}
0 \\
0 \\
d_{1}
\end{array}\right] \quad(2) \\
P_{2}=P_{1}
\end{gathered}
$$

$$
\begin{gathered}
P_{3}=\left[\begin{array}{c}
l_{1} c_{1} c_{2} \\
l_{1} s_{1} c_{2} \\
d_{1}-l_{1} s_{2}
\end{array}\right] \quad(4) \\
P_{4}=\left[\begin{array}{c}
d c_{1} c_{23}-l_{4} c_{1} s_{23}+l_{1} c_{1} c_{2} \\
d s_{1} c_{23}-l_{4} s_{1} s_{23}+l_{1} s_{1} c_{2} \\
d_{1}-d s_{23}-l_{4} c_{23}-l_{1} s_{2}
\end{array}\right]
\end{gathered}
$$

En robótica para simplificar se usa la notación $c_{i}=\cos \left(\theta_{i}\right), \quad s_{i}=\sin \left(\theta_{i}\right), \quad c_{i j}=\cos \left(\theta_{i}+\theta_{j}\right) \quad \mathrm{y}$ $s_{i j}=\sin \left(\theta_{i}+\theta_{j}\right), \quad$ donde $\quad i=1,2, \ldots, n \quad$ y $\quad \theta_{i}, \theta_{j}$ representan el número y las variables articulares del robot.

\section{Cinemática directa de velocidad}

La cinemática directa de velocidad determina la velocidad del efector-final a partir de las velocidades de las variables de cada articulación. Para obtener este modelo se debe definir la matriz jacobiana del manipulador. Esta puede considerarse como la versión vectorial de la derivada ordinaria de una función escalar. 11,12.

La cinemática directa de velocidad está definida por:

$$
V=\frac{d p_{i}(q)}{d t}=\frac{\partial p_{i}(q)}{\partial q} \frac{d q}{d t}=J p_{i}(q) \dot{q}
$$

Donde $J p_{i}$ es el Jacobiano respecto de cada una de las posiciones articulares del robot, $q \in \mathfrak{R}^{n}$ es el vector de posiciones articulares, $\dot{q} \in \mathfrak{R}^{n}$ es el vector de velocidades articulares. El Jacobiano de cualquier estructura de un robot manipulador es una matriz que relaciona las velocidades de movimiento de las articulaciones y las del efector-final del robot y pueden ser calculadas por la Ec. (6).

Para la velocidad $V_{1}$ tenemos: 


$$
V_{1}=\left[\begin{array}{lll}
0 & 0 & 0 \\
0 & 0 & 0 \\
0 & 0 & 0
\end{array}\right] \cdot\left[\begin{array}{l}
\dot{q}_{1} \\
\dot{q}_{2} \\
\dot{q}_{3}
\end{array}\right]
$$

Para la velocidad $V_{2}$ tenemos:

$$
V_{2}=V_{1}
$$

Para la velocidad $V_{3}$ tenemos:

$$
V_{3}=\left[\begin{array}{ccc}
-l_{1} s_{1} c_{2} & -l_{1} c_{1} s_{2} & 0 \\
l_{1} c_{1} c_{2} & -l_{1} s_{1} s_{2} & 0 \\
0 & -l_{1} c_{2} & 0
\end{array}\right] \cdot\left[\begin{array}{c}
\dot{q}_{1} \\
\dot{q}_{2} \\
\dot{q}_{3}
\end{array}\right]
$$

Para $V_{4}$ tenemos:

$$
V_{4}=\left[\begin{array}{lll}
J p_{4(11)} & J p_{4(12)} & J p_{4(13)} \\
J p_{4(21)} & J p_{4(22)} & J p_{4(23)} \\
J p_{4(31)} & J p_{4(32)} & J p_{4(33)}
\end{array}\right] \cdot\left[\begin{array}{c}
\dot{q}_{1} \\
\dot{q}_{2} \\
\dot{q}_{3}
\end{array}\right]
$$

Con:

$$
\begin{aligned}
& J p_{4(11)}=-d s_{1} c_{23}+l_{4} s_{1} s_{23}-l_{1} s_{1} c_{2} \\
& J p_{4(12)}=-d c_{1} s_{23}-l_{4} c_{1} c_{23}-l_{1} c_{1} s_{2} \\
& J p_{4(13)}=-d c_{1} s_{23}-l_{4} c_{1} c_{23} \\
& J p_{4(21)}=l_{1} c_{2} c_{1}-l_{4} c_{1} s_{23}+d c_{1} s_{23} \\
& J p_{4(22)}=-l_{1} s_{2} s_{1}-l_{4} s_{1} c_{23}-d s_{1} s_{23} \\
& J p_{4(23)}=-l_{4} s_{1} c_{23}-d s_{1} s_{23} \\
& J p_{4(31)}=0 \\
& J p_{4(32)}=-d c_{23}+l_{4} s_{23}-l_{1} c_{2} \\
& J p_{4(33)}=-d c_{23}+l_{4} s_{23}
\end{aligned}
$$

Después de haber obtenido la cinemática directa del "Robot Clony", ahora es posible obtener la posición del efector-final desde cada uno de los ángulos de las articulaciones $q_{1}, q_{2}$ y $q_{3}$.

\section{Cinemática inversa de posición}

La cinemática inversa trata el problema de encontrar los ángulos de las articulaciones requeridas para producir una cierta posición deseada y la orientación del efector-final. Encontrar la solución de la cinemática inversa para un manipulador puede ser una tarea muy difícil. Generalmente son ecuaciones no lineales. Sin embargo, los casos especiales tienen una solución de forma cerrada y se pueden resolver. El desarrollo de la cinemática inversa para el robot "Clony" se utiliza el método geométrico para encontrar la solución de la posiciones articulares $\theta_{1}, \theta_{2}$ y $\theta_{3}$ teniendo en cuenta la Figura 8 es fácil ver que la solución para $\theta_{1}$ es:

$$
\theta_{1}=A \tan 2\left(p_{w y}, p_{w x}\right)
$$

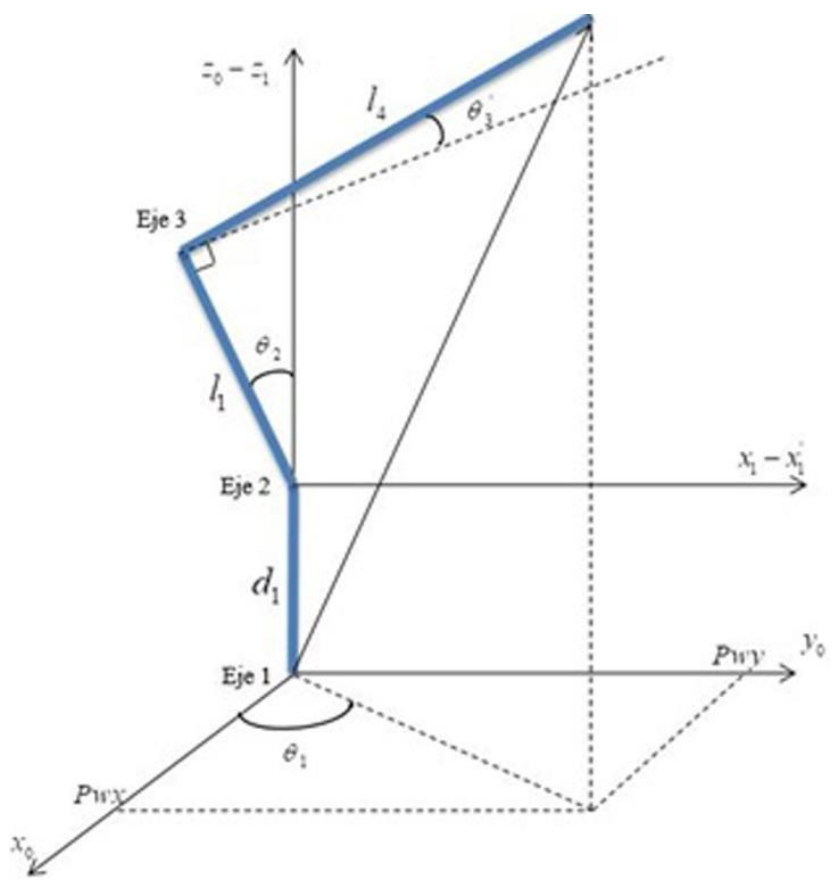

Figura 8. Análisis geométrico de la estructura del robot en $3 D$.

$$
\text { (Elaboración Propia) }
$$

Una vez obtenida $\theta_{1}$ el problema de la cinemática inversa se reduce a una estructura planar como se muestra en la Figura 9 , y es posible escribir:

$$
\begin{gathered}
p_{w x 1}=\sqrt{p_{w x}^{2}+p_{w y}^{2}} \\
p_{w z 1}=p_{w z}-d_{1}
\end{gathered}
$$




$$
\begin{aligned}
& p_{w y 1^{\prime}}=p_{w y 1} \\
& p_{w z 1^{\prime}}=p_{w z 1}
\end{aligned}
$$

\section{y}

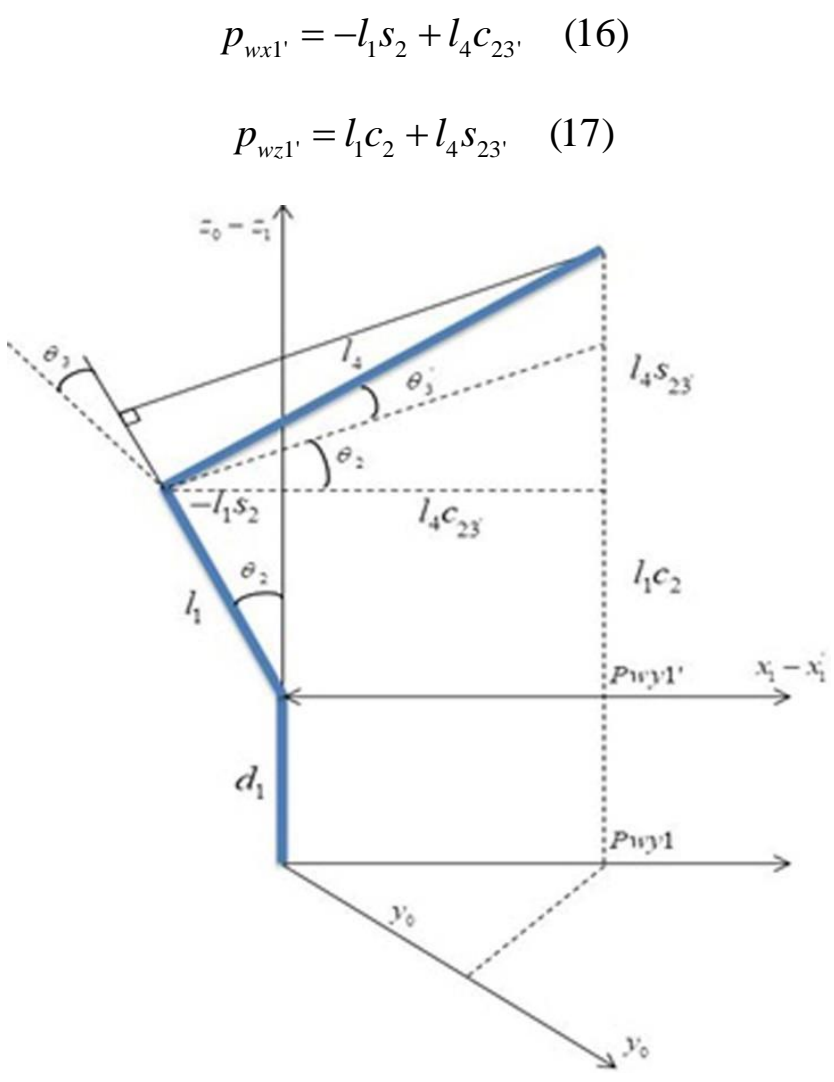

Figura 9. Análisis geométrico de la estructura del robot planar.

\section{(Elaboración Propia)}

Elevando al cuadrado y sumando las Ecs. (16) y (17) el resultado es:

$$
p_{w \times 1^{\prime}}^{2}+p_{w z 1^{\prime}}^{2}=l_{4}^{2}+l_{1}^{2}+2 l_{1} l_{4} s_{3^{\prime}}
$$

Lo que da:

$$
s_{3^{\prime}}=\frac{p_{w x 1^{\prime}}^{2}+p_{w z 11^{\prime}}^{2}-l_{4}^{2}-l_{1}^{2}}{2 l_{1} l_{4}}
$$

Estableciendo $c_{3}= \pm \sqrt{1-s_{3^{\prime}}^{2}}$, la solución para $\theta_{3}^{\prime}$ será:

$$
\begin{aligned}
& \theta_{3}^{\prime}=-A \tan 2\left(s_{3^{\prime}}, c_{3^{\prime}}\right) \\
& \theta_{3}=\theta_{3}^{\prime}+A \tan \left(\frac{d}{l_{4}}\right)
\end{aligned}
$$

Usando $\theta_{3}^{\prime}$ en Ecs. (16) y (17), resulta un Sistema de 2 ecuaciones con 2 incógnitas $s_{1}$ y $c_{1}$.

$$
\begin{gathered}
p_{w x 1^{\prime}}=-l_{1} s_{2}+l_{4}\left(c_{2} c_{3^{\prime}}-s_{2} s_{3^{\prime}}\right) \\
p_{w z 1^{\prime}}=l_{1} c_{2}+l_{4}\left(s_{2} c_{3^{\prime}}-c_{2} s_{3^{\prime}}\right)
\end{gathered}
$$

Resolviendo para $s_{2}$ y $c_{2}$ se obtiene:

$$
\begin{gathered}
s_{2}=\frac{-p_{w x 1^{1}}\left(l_{1}+l_{4} s_{3^{\prime}}+p_{w z 1^{1}}\right)}{l_{1}^{2}+l_{4}^{2} c_{3^{\prime}}^{2}+2 l_{1} l_{4^{\prime}} s_{3^{\prime}}+l_{4}^{2} s_{3^{\prime}}^{2}} \\
c_{2}=\frac{p_{w x x^{\prime}}\left(l_{4} c_{3^{\prime}}\right)+p_{w z 1^{\prime}}\left(l_{1}+l_{4} s_{3^{\prime}}\right)}{l_{1}^{2}+l_{4}^{2} c_{3^{\prime}}^{2}+2 l_{1} l_{4} s_{3^{\prime}}+l_{4}^{2} s_{3^{\prime}}^{2}}
\end{gathered}
$$

Y la solución para $\theta_{2}$ será:

$$
\theta_{2}=-A \tan \left(\frac{s_{2}}{c_{2}}\right)
$$

\section{Resultados}

Los resultados experimentales presentados son del robot manipulador de tres grados de libertad, (ver Figura 4). La validación del modelo cinemático se realizó sin aplicar una señal de entrada permitiendo el libre movimiento del robot manualmente, teniendo una captura de los datos de cada una de las articulaciones del robot. Los datos reales se comparan con el modelo cinemático inverso de posición utilizando los modelos de posición (11), (21) y (25) mostrados en la sección III. El diagrama de bloques para validar el modelo cinemático se muestra en la Figura 10. Los parámetros físicos del robot manipulador se muestran en la Tabla 12.

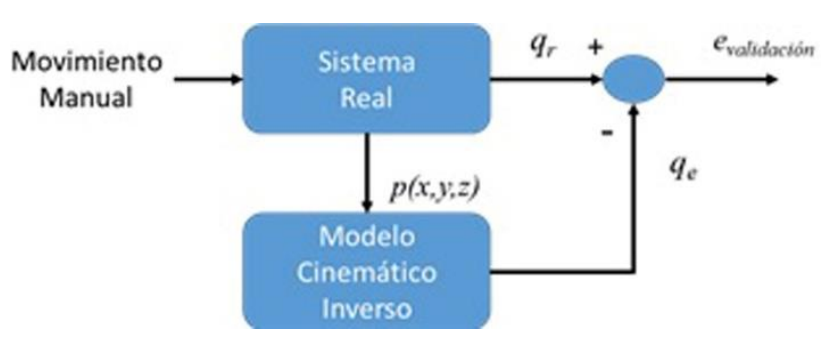


Figura 10. Diagrama a bloques para validación de modelo cinemático.

(Elaboración Propia)

Donde $p(x, y, z)$ es la posición del efecto-final del sistema real, $q_{r} \in \mathfrak{R}^{3}$ es la posición real del robot, $q_{e} \in \mathfrak{R}^{3}$ es la posición estimada del modelo cinemático inverso, $e_{\text {validación }} \in \mathfrak{R}^{3}$ es el error de validación definido por:

$$
e_{\text {validación }}=q_{r}-q_{e}
$$
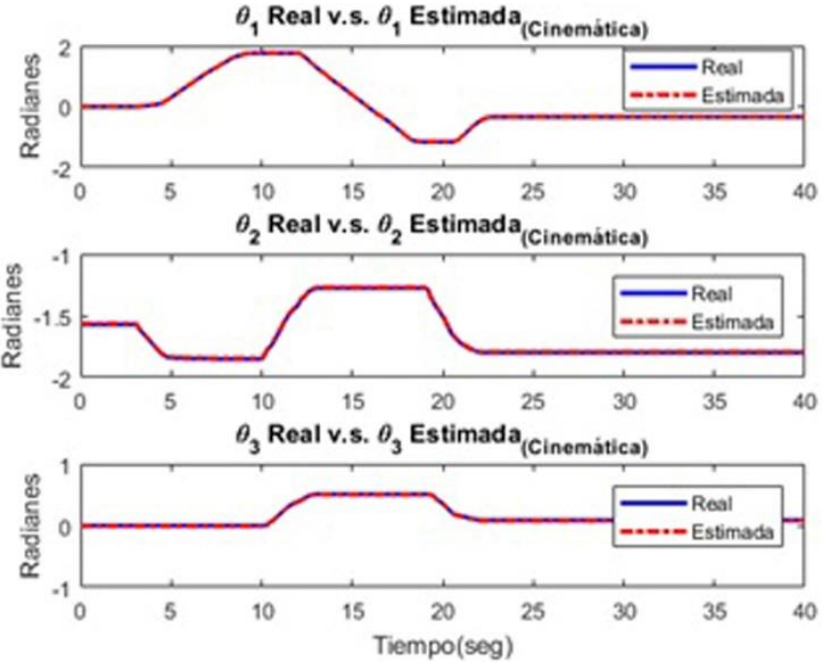

Figura 11. Validación del modelo cinemático.

(Elaboración Propia)

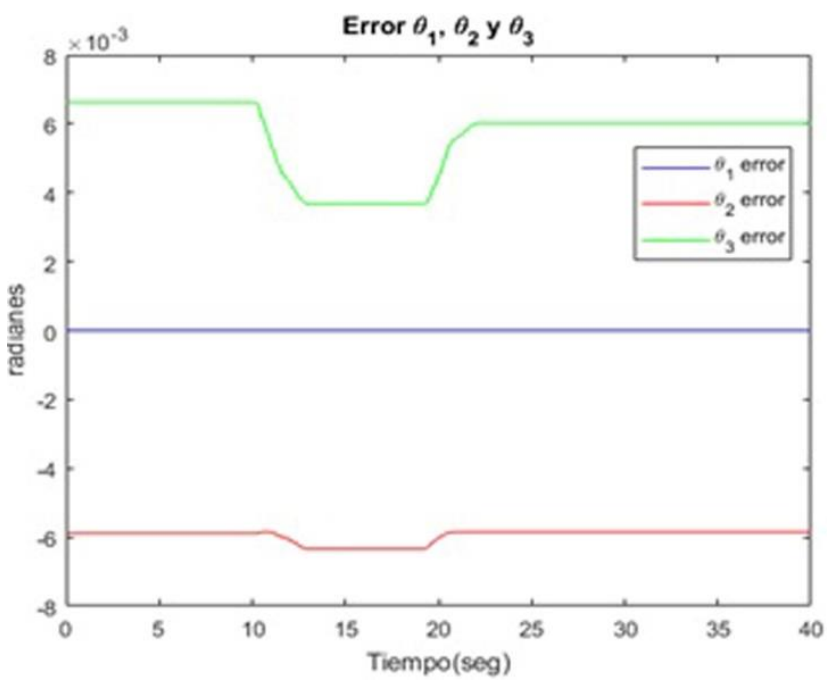

Figura 12. Error de validación.

(Elaboración Propia)

Los errores generados por cada articulación mostrados en la Figura 12, en donde se obtuvo un error de $0 \mathrm{rad}$, equivalente a $\left(0^{\circ}\right)$ aproximadamente para la articulación $\theta_{1}$, el error de estimación de $\theta_{2}$ se obtuvo un máximo error de $0.006637 \mathrm{rad}$, que es el equivalente a $\left(0.380^{\circ}\right)$ y un mínimo de $0.003669 \mathrm{rad}$, equivalente a $\left(0.210^{\circ}\right)$ y por último para la articulación $\theta_{3}$ se obtiene un máximo error de $-0.006345 \mathrm{rad}$, equivalente a $\left(-0.363^{\circ}\right)$ y un mínimo error de $-0.005892 \mathrm{rad}$, equivalente a $\left(-0.337^{\circ}\right)$. 


\section{Conclusiones}

- Se diseñó y se construyó un prototipo de robot manipulador de 5 barras y tres grados de libertad similar al robot industrial IRB 1400 de ABB con fines de investigación, mediante el uso de la metodología de Nigel Cross, la cual es una forma simple y racional para lograr un buen producto. EI prototipo puede ser utilizado para realizar prácticas de programación de robots, análisis estructural y de mecanismos.

- Los materiales de bajo costo como el nylamid, nylacero y aluminio presentan buenas propiedades mecánicas para el desarrollo de prototipos como el robot manipulador.

- El modelo cinemático obtenido del robot manipulador propuesto es apropiado para realizar simulaciones reales del robot, ya que el error de validación es aproximado a cero. También puede ser utilizado para investigación, para probar algoritmos de control de posición espacial. Los trabajos futuros a realizar son obtener el modelo matemático que incluya toda la dinámica del robot, y el control de seguimiento de trayectorias.

\section{Referencias}

[1] Cross N., "Engineering design methods: strategies for product design", John Wiley \& Sons, LTD. England, 3rd Ed., 2000.

[2] Giesecke F., Hill I., Spencer H., Mitchell A., Dygdon J., Novak J., y Lockhart S., "Dibujo técnico con gráficas de ingeniería", PEARSON, México, 14 Ed., 2013.

[3] Adueza L., Aguirre I., "Diseño de un manipulador robótico con tres grados de libertad para fines educativos". Revista Ciencia e Ingeniería. ISSN 1316-7081, Vol. 30, No. 1, pp.3-14, diciembre-marzo, 2009.

[4] Craig J., "Introduction to Robotics, Machanics and Control", AddisonWesley, USA, 2nd Ed., 1989.

[5] Reyes F., "Robótica. Control de robots manipuladores", Alfaomega, México, $1^{\circ}$ Ed., 2011.

[6] Barrientos A., Peñín L., Balaguer C. y Aracil R., "Fundamentos de robótica”, McGraw Hill, Madrid España, 2007.

[7] Urbalejo A., Jacobo E., Jiménez E., Quintero J. A., López J. R., Castro J. C., "Simulación, Control y Contrucción de un Robot Paralelo de 5 Barras y 6 GDL para Propositos Didacticos", $10^{\circ}$ Congreso Nacional de Mecatrónica., pp. 220-225 Noviembre 3 y 4, 2011.

[8] Urbalejo A., Cuenca F., Jiménez E., Jacobo E., Castro J. C., Velarde O., "Modelación y Simulación de un Robot Paralelo de 5 Barras y 6 GDL usando Matrices Homogéneas", $9^{\circ}$ Congreso Nacional de Mecatrónica., pp. 220-225 Octubre 13 -15, 2010, Puebla, Pue.

[9] Delfín J. "Análisis cinemático de un robot paralelo planar de tres grados de libertad tipo RRR”. México. (2004). Tesis de Maestría en Ingeniería en Mecánica. UNAM, Facultad de Ingeniería, División de Estudios de Posgrado, Sección Mecánica.

[10] Spong Mark W. "Robot Dynamic and Control", 1989.

[11] Fuente J.D, Santiago J, Román A, Dumitrache C, Casasanto D. "Handbook on Robotics". Siciliano, editor. Vol. 25. Berling: Springer; 2014., pp. 1682-1690.

[12] Cardoso E., Fernández A., Marrero S., Guardado P., "Modelos cinemático y dinámico de un robot de cuatro grados de libertad", RIELAC Vol.38, No.3, pp. 56-75, La Habana Sep.-Dic. 2017., ISSN 1815-5928.

\section{Anexo A}

\section{Introducción a las matrices}

Definición A.1 (Matrices) Una matriz A es un arreglo rectangular de números:

$$
A=\left(\begin{array}{cccc}
a_{11} & a_{12} & \cdots & a_{1 n} \\
a_{21} & a_{22} & \cdots & a_{2 n} \\
\vdots & \cdots & \ddots & \vdots \\
a_{m 1} & a_{m 1} & \cdots & a_{m n}
\end{array}\right)
$$

Si una matriz tiene $m$ filas y $n$ columnas, se dice que su tamaño es $m$ por $n$ (se escribe $m \times n$ ). Una matriz $n \times n$ se llama matriz cuadrada de orden $n$.

El término $a_{i j}$ representa el elemento de la $i$ - ésima fila y la $j$-ésima columna de una matriz A de tamaño $m \times n$ ; con esto, una matriz $A, m \times n$, se escribe en la forma $A=\left(a_{i j}\right) m \times n$, o simplemente $A=\left(a_{i j}\right)$ Una matriz $A, 1 \times 1$, es sólo un escalar (un número o una función).

Definición A.2 (lgualdad de matrices) Dos matrices A y B de $m \times n$, son iguales si y solo si $a_{i j}=b_{i j}$ para toda $i$ y $j$.

Definición A.3 (Matriz columna) Una matriz columna $X$ es cualquier matriz con $n$ filas y una columna: 


$$
X=\left(\begin{array}{c}
x_{11} \\
x_{21} \\
\vdots \\
x_{n 1}
\end{array}\right)=\left(x_{n 1}\right) \quad n \times 1
$$

Una matriz columna se llama también vector columna o simplemente vector.

Definición A.4 (Producto de matrices por escalares) Si $k$ es un escalar y $A$ una matriz $m \times n$, el producto de $k$ por $A$ es una nueva matriz que se define de la siguiente manera:

$$
k A=\left(\begin{array}{cccc}
k a_{11} & k a_{12} & \cdots & k a_{1 n} \\
k a_{21} & k a_{22} & \cdots & k a_{2 n} \\
\vdots & \cdots & \ddots & \vdots \\
k a_{m 1} & k a_{m 1} & \cdots & k a_{m n}
\end{array}\right)=\left(k a_{i j}\right) m \times n
$$

Donde $k$ es un escalar; es decir, un número o una función.

Definición A.5 (Derivada de una matriz de funciones) $\mathrm{Si}$ $A(t)=\left(a_{i j}(t)\right)$ es una matriz $m \times n$ cuyos elementos son funciones diferenciables en un intervalo común, entonces se define la derivada de $A(t)$ como la matriz cuyos elementos son las derivadas de los elementos de $A(t)$. Es decir:

$$
\frac{d A}{d t}(t)=\left(\frac{d a_{i j}}{d t}(t)\right) \quad m \times n
$$

\section{Matriz Jacobiana}

Sea $f$ una función diferenciable de un campo vectorial $n$-dimensional a otro campo vectorial $m$ - dimensional $f(x): \mathbb{R}^{n} \rightarrow \mathbb{R}^{m}$, con $x \in \mathbb{R}^{n}$. Las derivadas parciales de las $m$ funciones pueden ser representadas por un arreglo matricial denominado matriz jacobina.

La matriz jacobiana, representada por $J \in \mathbb{R}^{m \times n}$, es una matriz formada por derivadas parciales de primer orden, la cual puede escribirse de la siguiente forma:

$$
J=\frac{\partial f(x)}{\partial x}=\left[\begin{array}{cccc}
\frac{\partial f_{1}}{\partial x_{1}} & \frac{\partial f_{1}}{\partial x_{2}} & \cdots & \frac{\partial f_{1}}{\partial x_{n}} \\
\frac{\partial f_{2}}{\partial x_{1}} & \frac{\partial f_{2}}{\partial x_{2}} & \cdots & \frac{\partial f_{2}}{\partial x_{n}} \\
\vdots & \cdots & \ddots & \vdots \\
\frac{\partial f_{m}}{\partial x_{1}} & \frac{\partial f_{m}}{\partial x_{2}} & \cdots & \frac{\partial f_{m}}{\partial x_{n}}
\end{array}\right]
$$

